\title{
Status quo of exercise participation among gastric cancer patients after radical gastrectomy and analysis of the influencing factors
}

\author{
Danfeng Gu ${ }^{1 \#}$, Yuan Qian ${ }^{1 \#}$, Yuling Yang ${ }^{2}$, Huihong Wang ${ }^{3}$, Lingyun Zhu ${ }^{4}$, Xiaoying Chai ${ }^{4}$, Yu Zhang ${ }^{4}$ \\ ${ }^{1}$ Nursing Department, Affiliated Hospital of Jiangnan University, Wuxi, China; ${ }^{2}$ Corresponding author; Department of Surgery, Affiliated Hospital \\ of Jiangnan University, Wuxi, China; ${ }^{3}$ Outpatient department, Affiliated Hospital of Jiangnan University, Wuxi, China; ${ }^{4}$ Departmentof nephrology, \\ Affiliated Hospital of Jiangnan University, Wuxi, China \\ Contributions: (I) Conception and design: Y Yang, D Gu; (II) Administrative support: Y Qian, H Wang, L Zhu; (III) Provision of study materials \\ or patients: H Wang, L Zhu, X Chai, Y Zhang; (IV) Collection and assembly of data: Y Qian, Y Yang, D Gu, Y Zhang; (V) Data analysis and \\ interpretation: Y Yang, Y Qian; (VI) Manuscript writing: All authors; (VII) Final approval of manuscript: All authors. \\ \#These authors contributed equally to this work. \\ Correspondence to: Yuling Yang. Department of Surgery, Affiliated Hospital of Jiangnan University, Wuxi, China. Email: yulingzxc@163.com.
}

\begin{abstract}
Backgroundk To investigate the status quo of participation in exercise among gastric cancer patients after radical gastrectomy and analyze the influencing factors.

Methods: Convenient sampling was used to conduct a questionnaire survey of 163 patients after radical gastric cancer surgery from January to December 2020. The survey content included general information, exercise participation, exercise knowledge, attitude, and social support. Descriptive statistics, single factor analysis, and multiple linear regression analysis were performed using Statistical Product and Service Solutions 24.0 (SPSS24.0, IBM, USA).

Results: After radical gastrectomy, the form of exercise that patients participated in was relatively simple. The average amount of exercise involved was $8.10 \mathrm{Mets}-\mathrm{h} / \mathrm{week}$, which was at the level of almost no exercise. Univariate analysis showed that differences in age, gender, education level, work status, main caregivers and sports knowledge, attitudes, and social support levels all led to different levels of exercise participation. Multiple linear regression analysis showed that the factors affecting the patient's level of participation in exercise included age, degree of self-care in life, attitude towards exercise after surgery, and level of social support.

Conclusions: The status quo of exercise participation among gastric cancer patients after radical gastrectomy is not ideal. In this study, we found that age, level of self-care in life, sports attitude, and level of social support were the main factors affecting the exercise participation of patients. Therefore, improving patients' self-care ability, exercise attitude, and increasing social support may play an important role in improving the status quo of patients' exercise participation after radical gastric cancer surgery.
\end{abstract}

Keywords: Postoperative gastric cancer; exercise; influencing factors; attitude; social support

Submitted Apr 14, 2021. Accepted for publication Jun 16, 2021.

doi: 10.21037/apm-21-1241

View this article at: https://dx.doi.org/10.21037/apm-21-1241

\section{Introduction}

Cancer is a major disease that affects the health of people worldwide. According to the latest research on the global burden of disease, gastric cancer has become one of the top six causes of death in the Asia-Pacific region (1). At 6 present, there are approximately 952,000 new cases of gastric cancer globally, with the number of new cases in China accounting for approximately half $(405,000)$, and 9 
the rates of morbidity increasing annually (2). However, with the advancement of medical technology, the survival rate of patients has increased substantially (3), which has led to widespread attention regarding the rehabilitation of patients after gastric cancer surgery. As tumor growth leads to gastrointestinal dysfunction and surgical treatments cause traumatic stress, most patients will have varying degrees of dysfunction following radical gastric cancer surgery. In particular, patients 6 months after surgery often have a high frequency of symptoms, such as abdominal distension, pain, loss of body mass, and fatigue (4), which may not only affect their physical and mental state, but also increase the burden on their families and society.

Preliminary studies have reported that traditional retreats are questionable, and exercise therapy and clinical exercise prescriptions have gradually been introduced into cancer care as an adjuvant therapy (5). A large number of studies at home and abroad have shown that proper and reasonable exercise can relieve symptoms of physical discomfort to a certain extent, improve the immunity of cancer patients, and alleviate fatigue and insomnia. It can also relieve patients' psychological problems and negative emotions, such as anxiety and depression, thus improving their quality of life (6).

However, current research mainly focuses on the benefits of exercise rehabilitation for patients after gastric cancer surgery (7), and there is a lack of systematic research to measure the level of exercise participation in patients after gastric cancer surgery. Due to the pathological complexity of the disease itself and a series of sequelae in patients after radical gastric cancer surgery, the factors affecting their level of participation in exercise may be more complicated.

The purpose of this study is to investigate the current status of exercise participation in patients after radical gastric cancer surgery based on the knowledge-attitudepractice pattern (8), and analyze its influencing factors, so as to provide guidance and reference for the targeted improvement of patients' exercise participation levels after radical gastric cancer surgery.

We present the following article in accordance with the SURGE reporting checklist (available at: https://dx.doi. org/10.21037/apm-21-1241).

\section{Methods}

\section{Objects}

Convenient sampling was used to conduct a questionnaire survey of patients after radical gastric cancer surgery in 58 the Binhu District community, Wuxi City from January to 59 December 2020. In this study, postoperative gastric cancer 60 patients refer to patients 6 months after radical gastric cancer $\quad 61$ surgery. These patients were in a relatively stable condition $\quad 62$ and should have been engaging in appropriate exercises to 63 improve physical function. The inclusion criteria were as 64 follows: (I) patients with pathologically diagnosed gastric 65 cancer; (II) patients that underwent radical gastric cancer 66 surgery, and the postoperative time was over 6 months; 67 (III) patients over 18 years of age; and (IV) patients with 68 a clear consciousness, and those able to understand and 69 cooperate with this study. The exclusion criteria were as 70 follows: (I) those who received radiotherapy, chemotherapy, 71 or hormone replacement therapy within 1 month prior 72 to survey; (II) those with distant tumor metastases; (III) 73 patients with severe cognitive dysfunction and mental 74 disorders; and (IV) patients with severe cardiovascular 75 diseases or any other exercise contraindications, who 76 are not suitable for exercise. In total, 170 postoperative 77 radical gastric cancer surgery patients were enrolled, and 78 163 questionnaires were collected, with an effective recovery 79 rate of $95.9 \%$. Informed consent was obtained from all the 80 patients enrolled in this study. All procedures performed in 81 this study involving human participants were in accordance 82 with the Declaration of Helsinki (as revised in 2013). The 83 study was approved by institutional ethics committee of the 84 Affiliated Hospital of Jiangnan University (No. LS2019043: the registration number of ethics board).

\section{Survey tool}

\section{General information}

The contents included socio-demographic data and diseaserelated data of patients following radical gastric cancer surgery. Socio-demographic data includes the patient's age, gender, education level, work status, etc.; and diseaserelated data includes the patient's tumor staging, number of sequelae, and degree of self-care. The degree of selfcare in life is measured using the Activity of Daily Living Scale (ADL), which comprises 10 items including eating, bathing, grooming, dressing, bowel control, toileting, bed and wheelchair transfer, walking on the ground, and stairs. Based on the patient's need for help (or lack thereof) and its degree, the ADL scale is divided into 4 grades of 15,10 , 5 , and 0 points, with a full score of 100 points. A score of 0 -20 points signifies extremely severe dysfunction, that is, an 83 84 85

9
1

3

4

5

6

67

9

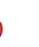

2

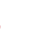

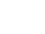

6

7

78

79


inability to take care of oneself at all; a score of $21-40$ points denotes severe dysfunction, which is an inability to take care of oneself; a score of 41-60 points signals moderate dysfunction, which means that the patient is able to take care of oneself basically; a score of 61-99 points signifies mild dysfunction, that is, an ability to take care of oneself almost completely; and 100 points denotes no dysfunction, which is an ability to take care of oneself completely.

\section{Status quo of exercise participation among patients with gastric cancer after radical gastrectomy}

The revised "Amateur Exercise Level Questionnaire" was used for the survey. The questionnaire was revised by Professor Kriska based on previous research results and the characteristics of mass sports in China, which is suitable for surveys of sports participation in all types of people. The questionnaire lists 40 sports items such as walking, running, mountain climbing, and biking. The respondents were required to select the items that have been done more than 10 times in the past 12 months, tick the items in the corresponding month, and fill in each item. The average exercise duration and the average frequency of exercises were recorded. The researcher then calculated the total amount of exercise engaged in by the patient based on the metabolic equivalent (MET) value of each sports event. The calculation formula was as follows: number of months $\times$ average number of monthly exercises $\times$ average exercise duration per time $\times$ MET/60 min/h/52 wk/year. According to the calculated total exercise volume (unit: Mets-h/week), the results could be divided into four levels: hardly exercise group (<10.0 Mets-h/week), low-level exercise group (10-19.9 Mets-h/week) week), mediumlevel exercise group (20-39.9 Mets-h/week), and high-level exercise group ( $\geq 40$ Mets-h/week).

Since the questionnaire used in this study was adapted from a foreign questionnaire, many of the include sports are not common in China. Therefore, we made appropriate modifications and conducted expert consultation. After two rounds of expert consultation, all experts agreed that this questionnaire was suitable to survey the level of participation in exercise of patients after radical gastric cancer surgery. After adjustment, the questionnaire retained 14 items. The content validity (I-CVI) of each item of the revised questionnaire was between 0.78 and 1 , and the overall content validity (S-CVI) of the questionnaire was
0.97 , indicating that the questionnaire had good validity.

Exercise knowledge, attitude, and social support level of patients after radical gastric cancer surgery

The knowledge-attitude-practice pattern proposes that knowledge is the basis of behavioral change, and belief is the driving force of behavioral change. Thus, only by understanding relevant health knowledge and establishing positive and correct beliefs is it possible to actively form healthy behaviors (8). By consulting the literature and combining the opinions of patients and related experts following radical gastric cancer surgery, the research team found that knowledge, attitudes, and social support related to exercise after radical gastric cancer surgery may have a significant influence on the patient's exercise participation level. Therefore, we designed the knowledge-attitudebehavior questionnaire of exercise of patients after radical gastrectomy for cancer. The patient's knowledge-attitudebehavior questionnaire conducts related investigations. This questionnaire includes three aspects: knowledge, attitude, and social support, with a total of 19 items. The total scores for each part of the questionnaire were 35 , 30 , and 30 , respectively. The criteria for evaluating the results were as follows: scores less than $60 \%$ of the total score were considered poor; scores between $60 \%$ and $80 \%$ were considered good, and scores greater than $80 \%$ were considered excellent. The content validity of the questionnaire was 0.95 , and the Cronbach's $\alpha$ coefficient was 0.94 , indicating that the questionnaire had good reliability and validity, and could be used as a credible tool to evaluate the exercise knowledge, attitude, and social support level of patients after radical gastric cancer surgery.

\section{Statistical analysis}

Statistical analyses were performed using Statistical Product and Service Solutions 24.0 (SPSS24.0, IBM, USA). Categorical variables were expressed as $\mathrm{n} / \%$, and compared by using chi-square test or Fisher' exact test. Continuous variables were expressed as mean \pm standard deviation or median (P25, P75) and compared using $\mathrm{t}$ test or MannWhitney $\mathrm{U}$ test. Univariate factor analysis and multiple linear regression analysis were used to identify the factors associated with exercise participation among gastric cancer patients after radical gastrectomy. $\mathrm{A} \mathrm{P}<0.05$ was considered 
Table 1 Socio-demographic data

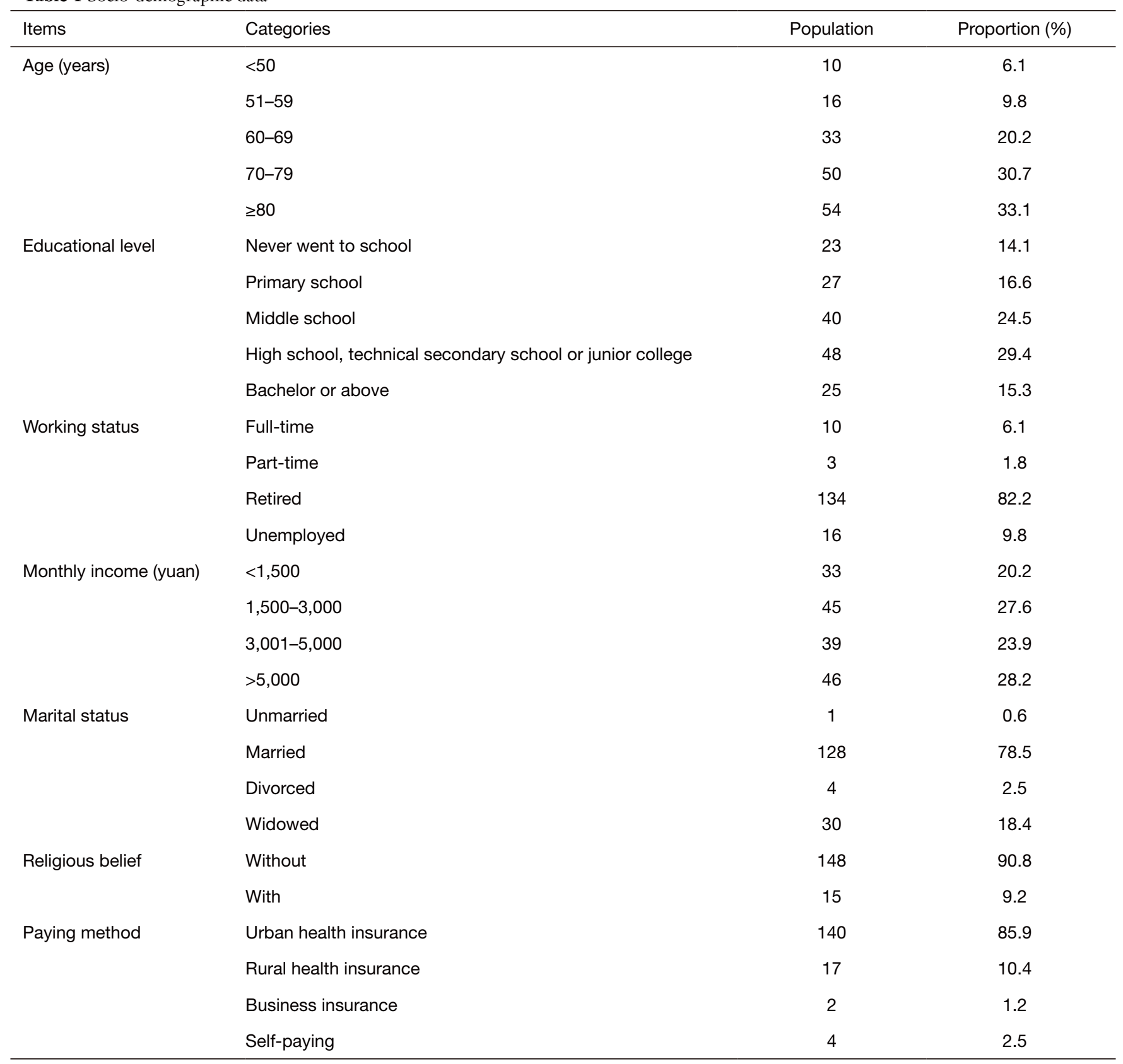

statistically significant.

\section{Results}

\section{General information of patients}

Socio-demographic data

The 163 survey subjects in this study included 93 males
(57.1\%) and 70 females (42.9\%), with an average age of 203 $(71.9 \pm 12.7)$ years (range, $32-95$ years). The details are 204 shown in Table 1.

Disease-related information

The shortest disease course of patients in this study was 6 months and the longest was 12 years; $82.8 \%$ of patients had other chronic diseases such as diabetes, hypertension, 210 
Table 2 Disease-related information

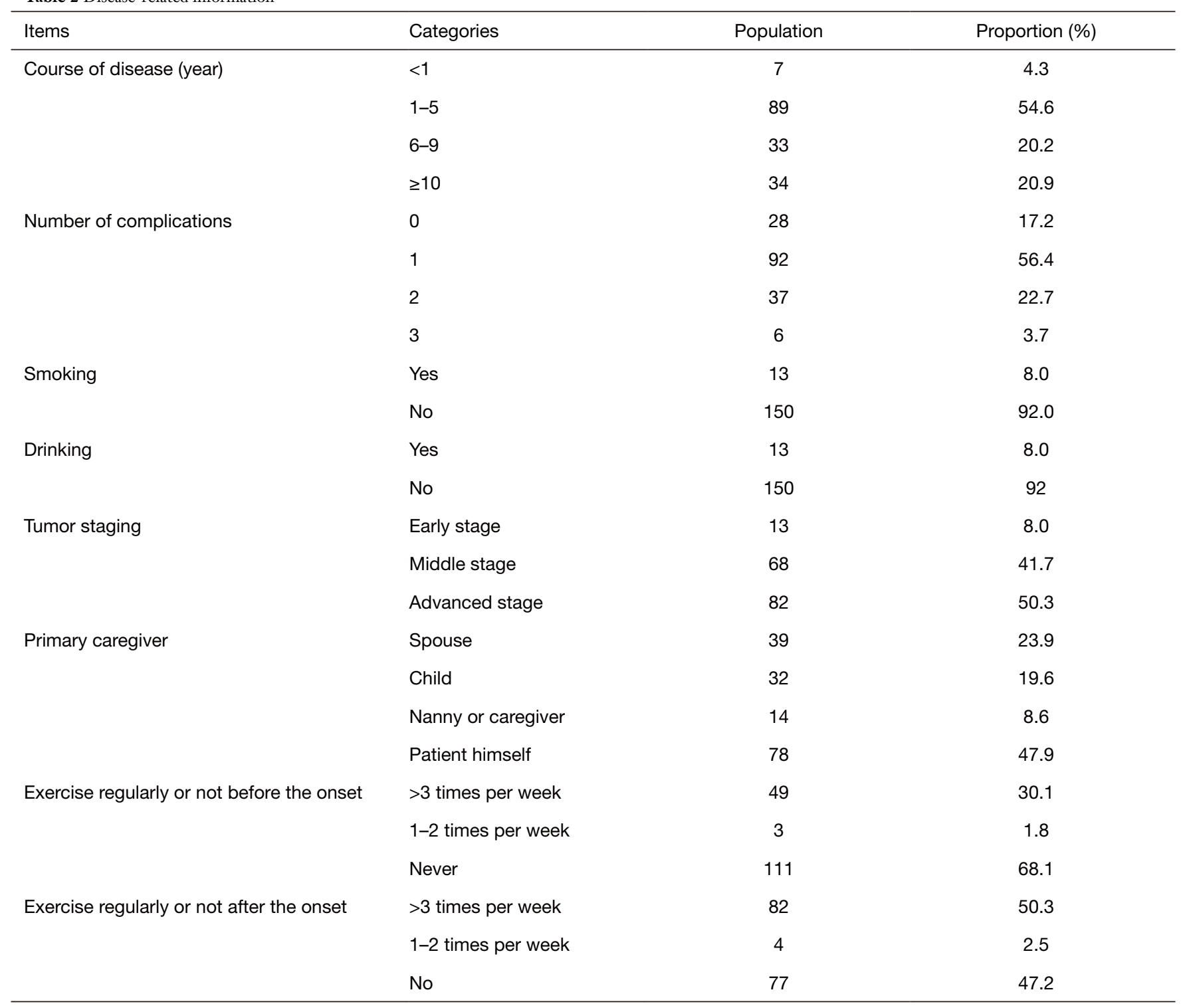

etc.; and $81 \%$ of patients had different degrees of dysfunction, including loss of appetite, pain, fatigue, and decreased physical fitness. The disease-related information is shown in Table 2.

\section{Self-care degree of life}

In this survey, $77.9 \%$ [127] patients were able to take care of themselves. Patients with mild, moderate, and severe life disorders accounted for $11.0 \%$ [18], 4.9\% [8], and 6.1\% [10] of patients, respectively.
Status quo of exercise participation 221

According to the results of the survey, it was found that the average amount of exercise for patients after radical gastric cancer surgery was $8.10 \mathrm{Mets}-\mathrm{h} /$ week, which was at the level of almost no exercise (Table 3).

In addition, our study found that the range of exercise options selected by patients was narrow and single. Except for walking, climbing stairs, and indoor activities, the number of people participating in other sports as less than 
Table 3 Exercise amount of patients after radical gastrectomy

\begin{tabular}{llcc}
\hline Items & Group & Population & Exercise amount (Mets-h/week), M (P25, P75) \\
\hline Exercise amount & Total & 163 & $8.1(2.4,18.8)$ \\
& Almost no exercise & 91 & $3.2(1.1,5.8)$ \\
& Low level of exercise amount & 39 & $15.5(12.9,18.1)$ \\
& Middle level of exercise amount & 25 & $28.7(23.3,32.6)$ \\
& High level of exercise amount & 8 & $53.9(44.9,76.9)$ \\
\hline
\end{tabular}

Table 4 The number and proportion of patients who participated in the three most common types of exercise after radical gastric cancer surgery

\begin{tabular}{lcc}
\hline Items & Population & Proportion (\%) \\
\hline Walking & 138 & 84.7 \\
Climbing stairs & 44 & 27.0 \\
$\begin{array}{l}\text { Indoor activities } \\
\text { (standing on tiptoes, kicking, etc.) }\end{array}$ & 34 & 20.9 \\
\hline
\end{tabular}

Table 5 The number and proportion of patients who participated in a different number of exercise types after radical gastric cancer surgery

\begin{tabular}{lcc}
\hline Number of exercise types & Population & Proportion (\%) \\
\hline 1 & 100 & 61.3 \\
2 & 50 & 30.7 \\
3 & 11 & 6.7 \\
$>3$ & 2 & 1.2 \\
\hline
\end{tabular}

$20 \%$. Approximately $92 \%$ of patients only participated in one or two sports. Tables 4 and 5 list the three most common types of sports that patients participated in, and the number and proportion of participants in different sports, respectively.

\section{Patient's exercise knowledge, attitude, and social support level}

The average scores of exercise knowledge, attitude, and social support of patients after radical gastric cancer surgery were 18,17 , and 17 , respectively. These scores were all less than $60 \%$ of the total score, which was at a poor level. The
Table 6 Exercise knowledge, attitude and social support scores of patients after radical gastric cancer surgery

\begin{tabular}{llll}
\hline \multirow{2}{*}{ Items } & Categories & $\begin{array}{c}\text { Mean score, } \\
\text { M (P25, P75) }\end{array}$ & Score/total $(\%)$ \\
\hline Knowledge & Total & $18(12.0,26.0)$ & 51.4 \\
& Poor & $13(10.0,16.0)$ & 37.1 \\
& Good & $24(22.0,25.8)$ & 68.6 \\
& Excellent & $31(30.0,33.0)$ & 88.6 \\
Attitude & Total & $17(13.0,22.0)$ & 56.7 \\
& Poor & $13(10.0,15.0)$ & 43.3 \\
& Good & $20(18.0,22.0)$ & 66.7 \\
& Excellent & $27(24.8,29.0)$ & 90.0 \\
Social support & Total & $17(13.0,21.0)$ & 48.6 \\
& Poor & $13(11.0,15.0)$ & 43.3 \\
& Good & $19(19.0,21.0)$ & 63.3 \\
& Excellent & $26(25.0,27.5)$ & 74.3 \\
\hline
\end{tabular}

specific scores and classifications are shown in Table 6.

Single factor analysis of exercise participation level

In order to analyze and compare the levels of exercise 248 participation in patients of different categories, a rank-sum 249 test was performed, with the items in the general data and 250 the level of exercise knowledge, attitude, and social support 251 after gastric cancer surgery used as independent variables, 252 and the amount of exercise used as the dependent variable. 253 As shown in Table 7, the differences in exercise participation 254 levels among patients with different ages, genders, education 255 levels, work status, number of strokes, main caregivers, and 256 
Table 7 Single factor analysis result of sports participation level

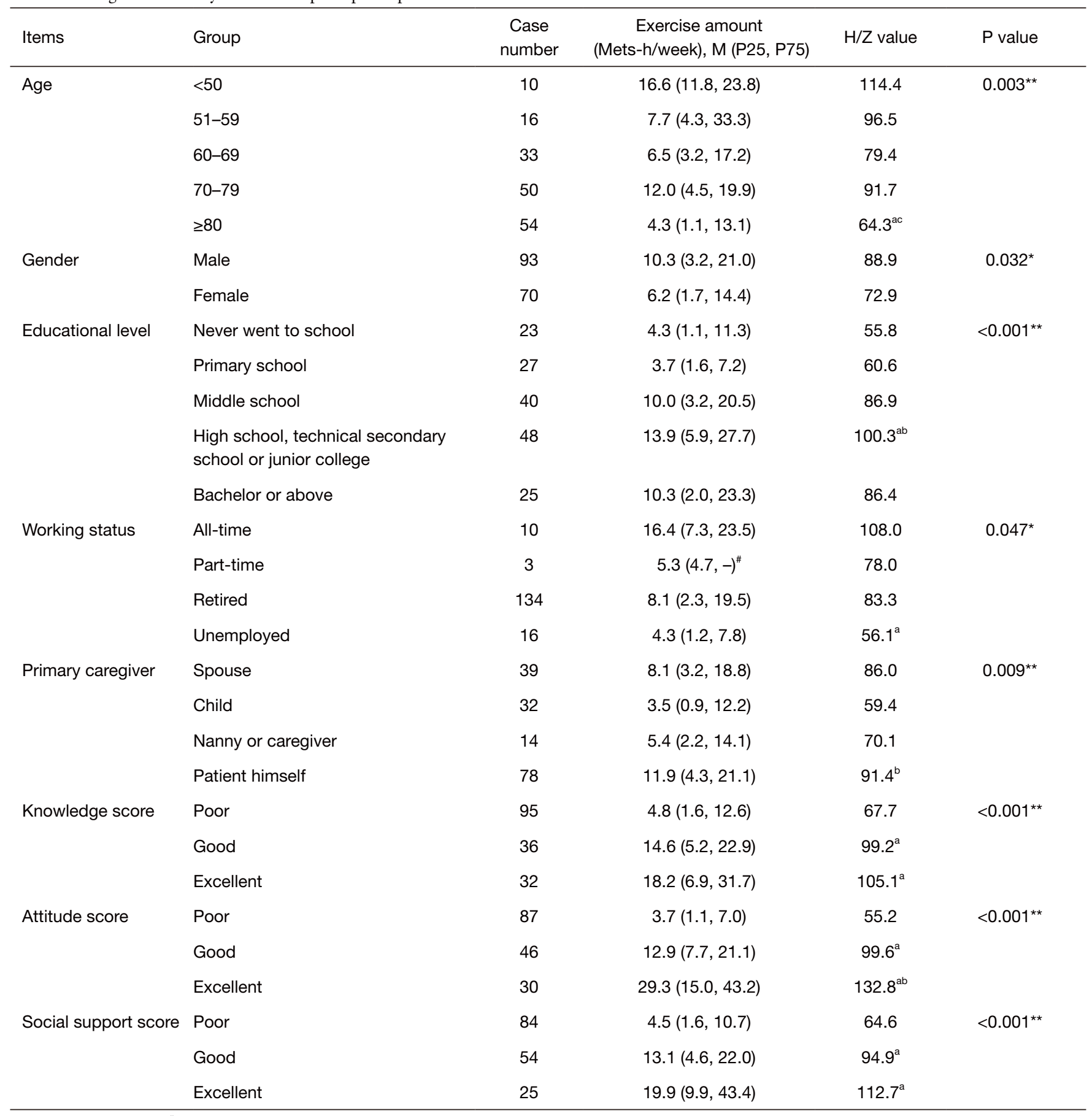

${ }^{\star} \mathrm{P}<0.05,{ }^{\star} \mathrm{P}<0.01$; "Since there were only three patients in this group, the data of P75 after analysis is indicated by "-"; adifferent from the first category of the item; ' different from the second category of the item; ' different from the fourth category of the item. 
Table 8 Multiple linear regression analysis of sports participation level

\begin{tabular}{lccccc}
\hline \multirow{2}{*}{ Independent variable } & $\beta$ & $\beta^{6}$ & $P$ & \multicolumn{2}{c}{$95 \%$ confidence interval } \\
\cline { 4 - 5 } Constant & -12.455 & & 0.260 & -34.221 & Lower limit \\
Age & -0.198 & -0.148 & 0.036 & -0.383 & -0.013 \\
Self-care level & 3.967 & 0.197 & 0.003 & 1.330 & 1.173 \\
Knowledge score & 1.592 & 0.571 & $<0.001$ & 0.003 \\
Social support score & 0.464 & 0.147 & 0.046 & 0.003 \\
\hline
\end{tabular}

different post-stroke exercise knowledge, attitudes, and social support levels were all statistically significant.

\section{Multi-factor analysis of sports participation level}

In order to further clarify the influencing factors of the level of exercise participation in patients after radical gastric cancer surgery, multiple linear regression analysis was performed, with exercise volume used as the dependent variable and the remaining items used as independent variables. Binary variables, grade variables and measurement data were directly entered into the equation with original values using the stepwise entry method (Table 8). According to the statistical results, a model was established according to the following formula: the patient's exercise amount = $-12.455-0.198 \times$ age $+3.967 \times$ self-care score $+1.592 \times$ attitude score $+0.464 \times$ social support score.

\section{Discussion}

In this study, based on the knowledge-attitude-practice pattern, existing questionnaires combined with selfdesigned questionnaires were used to investigate the level of exercise participation among 163 patients after radical gastric cancer surgery and analyze the influencing factors.

\section{Status quo of exercise participation of patients after radical gastric cancer surgery}

In the survey, we found that the overall exercise level of patients after radical gastric cancer surgery was at the level of almost non-exercise, which is consistent with the poor exercise participation level of patients after radical gastric cancer surgery that has been reflected in other studies (9). A lack of exercise will slow the patient's recovery progress and increase the risk of disease recurrence (10), which deserves our attention.

In addition, the type of exercise type that postoperative patients engaged in was relatively simple, mostly exercises that are low in difficulty, mild in intensity, and do not require excessive motor skills (such as climbing stairs and walking). On one hand, this suggests that these patients may lack motor skills. Furthermore, sports that are not similar to daily activities, such as yoga, Tai Chi, and ball sports, usually require specific learning to master sports skills, and some patients may lack these learning opportunities, resulting in lower participation. Studies have shown that exercises that require coordination of all parts of the body are better than exercises that require only a part of the limbs. To achieve the recommended amount of exercise, patients can engage in either short-term high-intensity exercise or long-term low-intensity exercise (11). Therefore, we recommend that patients participate in yoga, Tai Chi, and other activities that require the participation of all parts of the body and are not high intensity.

\section{Factors affecting the level of exercise participation of patients after radical gastric cancer surgery}

Univariate analysis showed that differences in age, gender, education level, work status, main caregivers, and exercise knowledge, attitudes, and social support levels after gastric cancer surgery all led to different levels of exercise participation. Given that there are many influencing factors in a single factor analysis, multiple linear regression analysis was performed to explore the factors that ultimately affect the patient's exercise participation level. It was found that age, degree of self-care in life, level of exercise attitude, and level of social support were the main factors affecting the level of exercise participation of patients after radical gastric 
cancer surgery.

\section{Age}

This survey found that age negatively affects the level of patient participation in sports. Younger patients need to bear the support of their families and the entire society, and their urgency and demand for rehabilitation are also higher (12). This is a strong motivation for young patients to engage in sports, and leads to a relatively high level of sports participation. In addition to the disease factors after radical gastric cancer surgery, aging itself will result in a series of physical functional degradations and various comorbidities, leading to reduced energy, limited limb function, and fear of exercise risks in patients, which will hinder their participation in exercise. Studies have shown that exercise can also improve limb function in elderly patients after radical gastric cancer surgery (10). Therefore, patients of all ages should be encouraged to actively participate in sports, and at the same time, exercise guidance and supervision should be strengthened to reduce exercise risks and improve patients' enthusiasm for exercise.

\section{Self-care level}

Multiple linear regression analysis also showed that the degree of self-care in life is an important factor affecting patients' participation in exercise after radical gastric cancer surgery, which is consistent with the results of research carried out at home and abroad. Patients with poorer selfcare level typically have more severe impairment of limb function and greater difficulty during exercise. Some patients with severely impaired self-care ability can only stay in bed or are sedentary for extended periods of time (9), which directly affects their level of participation in sports. The loss of selfconfidence and various dysfunctions caused by the substantial damage to the basic condition of the body will also cause patients to be unwilling to participate in sports, and may even lead to the idea of self-destruction (7).In addition, the poor self-care of patients will increase the burden on caregivers, making them prone to fatigue and depression, etc., which is not conducive to their ability to provide support and supervision of patients' participation in sports.

\section{Exercise attitude after radical gastric cancer surgery}

Patients with better exercise attitude have higher levels of exercise participation. Sullivan et al. (13) pointed out that a patient's healthy attitude is crucial to changing unhealthy behaviors. Patients with a good attitude towards exercise, even without the supervision of others, will lead to a higher level of exercise participation. Some scholars believe that if patients have a better attitude towards exercise, they will exhibit better exercise compliance, which will lead to greater exercise participation (3). It is generally believed that patients with a better attitude towards exercise will demonstrate a superior ability to overcome difficulties, which is conducive to the patient's persistence in exercise. Some patients with good exercise attitudes also set higher rehabilitation goals for themselves. Moreover, Deci et al.'s self-determination theory posits that the goals set by individuals for results are conducive to maintaining healthy behaviors (14).

Exercise and social support after gastric cancer surgery Social support refers to the supportive resources that individuals obtain from others or social networks, which can help them cope with problems in life (15). The improvement of sports participation requires not only the cooperation of patients, but also the help and encouragement of family members and the support of related sports facilities (16). In this survey, we found that the overall level of social support of the patients was poor, which will indirectly reduce the patient's level of participation in sports. The presence of a dedicated person to guide the knowledge and skills of sports after radical gastric cancer surgery will effectively improve the patient's level of sports participation (10).

\section{Limitation}

The number of patient samples in this study is too small, and a large sample study should be added for verification.

\section{Conclusions}

The current status quo of participation in exercise among patients after radical gastric cancer surgery is not ideal; their levels of exercise are low, and their exercise methods are relatively simple. The level of participation in exercise is primarily affected by the patient's age, degree of self-care in life, attitude towards exercise after surgery, and level of social support. Therefore, strengthening early rehabilitation training for patients after radical gastric cancer surgery, improving patients' self-care ability, vigorously promoting the importance of exercise after radical gastric cancer surgery, and mobilizing families and society to actively participate in and help patients undergoing radical gastric 
cancer surgery to engage in a reasonable amount of exercise will improve the level of patient participation in exercise.

\section{Acknowledgments}

Funding: This study is funded by the Wuxi Municipal Health Commission (No. MS201945).

\section{Footnote}

Reporting Checklist: The authors have completed the SURGE reporting checklist. Available at https://dx.doi.org/ apm-21-1241

Data Sharing Statement: Available at https://dx.doi.org/apm21-1241

Conflicts of Interest: All authors have completed the ICMJE uniform disclosure form (available at https://dx.doi.org/ apm-21-1241). The authors have no conflicts of interest to declare.

Ethical Statement: The authors are accountable for all aspects of the work in ensuring that questions related to the accuracy or integrity of any part of the work are appropriately investigated and resolved. All procedures performed in this study involving human participants were in accordance with the Declaration of Helsinki (as revised in 2013). The study was approved by institutional ethics committee of the Affiliated Hospital of Jiangnan University (No. LS2019043: the registration number of ethics board) and informed consent was taken from all the patients.

Open Access Statement: This is an Open Access article distributed in accordance with the Creative Commons Attribution-NonCommercial-NoDerivs 4.0 International License (CC BY-NC-ND 4.0), which permits the noncommercial replication and distribution of the article with the strict proviso that no changes or edits are made and the original work is properly cited (including links to both the formal publication through the relevant DOI and the license). See: https://creativecommons.org/licenses/by-nc-nd/4.0/.

\section{References}

1. GBD 2013 Mortality and Causes of Death Collaborators. Global, regional, and national age-sex specific all-cause and cause-specific mortality for 240 causes of death, 1990-
2013: a systematic analysis for the Global Burden of

Disease Study 2013. Lancet 2015;385:117-71.

2. Wang C, Zhang J, Cai M, et al. DBGC: A Database of Human Gastric Cancer. PLoS One 2015;10:e0142591.

3. Waldum HL, Fossmark R. Types of Gastric Carcinomas. Int J Mol Sci 2018;19:4109.

4. Machlowska J, Baj J, Sitarz M, et al. Gastric Cancer: Epidemiology, Risk Factors, Classification, Genomic Characteristics and Treatment Strategies. Int J Mol Sci 2020;21:4012.

5. Neil-Sztramko SE, Medysky ME, Campbell KL, et al. Attention to the principles of exercise training in exercise studies on prostate cancer survivors: a systematic review. BMC Cancer 2019;19:321.

6. Huerta JM, Chirlaque MD, Molina AJ, et al. Physical activity domains and risk of gastric adenocarcinoma in the MCC-Spain case-control study. PLoS One 2017;12:e0179731.

7. Maltser S, Cristian A, Silver JK, et al. A Focused Review of Safety Considerations in Cancer Rehabilitation. PM\&R 2017;9:S415-28.

8. Lau J, Lim TZ, Jianlin Wong G, et al. The health belief model and colorectal cancer screening in the general population: A systematic review. Prev Med Rep 2020;20:101223.

9. Gjerset GM, Fosså SD, Courneya KS, et al. Exercise behavior in cancer survivors and associated factors. J Cancer Surviv 2011;5:35-43.

10. Cortes J, Hudgens S, Twelves C, et al. Health-related quality of life in patients with locally advanced or metastatic breast cancer treated with eribulin mesylate or capecitabine in an open-label randomized phase 3 trial. Breast Cancer Res Treat 2015;154:509-20.

11. Billinger SA, Arena R, Bernhardt J, et al. Physical activity and exercise recommendations for stroke survivors: a statement for healthcare professionals from the American Heart Association/American Stroke Association. Stroke 2014;45:2532-53.

12. Schulz R, Beach SR, Czaja SJ, et al. Family Caregiving for Older Adults. Annu Rev Psychol 2020;71:635-59.

13. Sullivan KA, Katajamaki A. Stroke Education: Promising Effects on the Health Beliefs of Those at Risk. Top Stroke Rehabil 2009;16:377-87.

14. Deci E. An interview with Dr. Edward L. Deci, codeveloper of self-determination theory. Interview by Paul Terry. Am J Health Promotion Ajhp 2013;27:TAHP2-7.

15. Karahan AY, Kucuksen S, Yilmaz H, et al. Effects of 
rehabilitation services on anxiety, depression, care-giving burden and perceived social support of stroke caregivers. Acta Medica 2014;57:68.

16. Wang TC, Tsai AC, Wang JY, et al. Caregiver-mediated intervention can improve physical functional recovery of patients with chronic stroke: a randomized controlled trial. $\quad 522$

Neurorehabilitation Neural Repair 2015;29:3.

523

524

(English Language Editor: A. Kassem)

Cite this article as: Gu D, Qian Y, Yang Y, Wang H, Zhu L, Chai X, Zhang Y. Status quo of exercise participation among gastric cancer patients after radical gastrectomy and analysis of the influencing factors. Ann Palliat Med 2021;10(6):6650-6660. doi: 10.21037/apm-21-1241 